\title{
Coupling Epitaxy, Chemical Bonding, and Work Function at the Local Scale in Transition Metal-Supported Graphene
}

\author{
Bo Wang, Marco Caffio, Catherine Bromley, Herbert Früchtl, \\ and Renald Schaub*
}

School of Chemistry, University of St. Andrews, St. Andrews, KY16 9ST, United Kingdom

Corresponding Author $\left(^{*}\right)$ : $\quad$ renald.schaub@st-andrews.ac.uk.

Resonance tunneling spectroscopy and density functional theory calculations are employed to explore local variations in the electronic surface potential of a single graphene layer grown on $\mathrm{Rh}(111)$. A work function modulation of $220 \mathrm{meV}$ is experimentally measured, indicating that the chemical bonding strength varies significantly across the supercell of the Moiré pattern formed when graphene is bonded to $\mathrm{Rh}(111)$. In combination with high-resolution images, which provide precise knowledge of the local atomic registry at the carbon metal interface, we identify experimentally, and confirm theoretically, the atomic configuration of maximum chemical bonding to the substrate. Our observations are at odds with reported trends for other transition metal substrates. We explain why this is the case by considering the various factors that contribute to the bonding at the graphene/metal interface.

\section{KEYWORDS}

Graphene, rhodium, chemical bonding, scanning tunnelling microscopy, density functional theory, work function
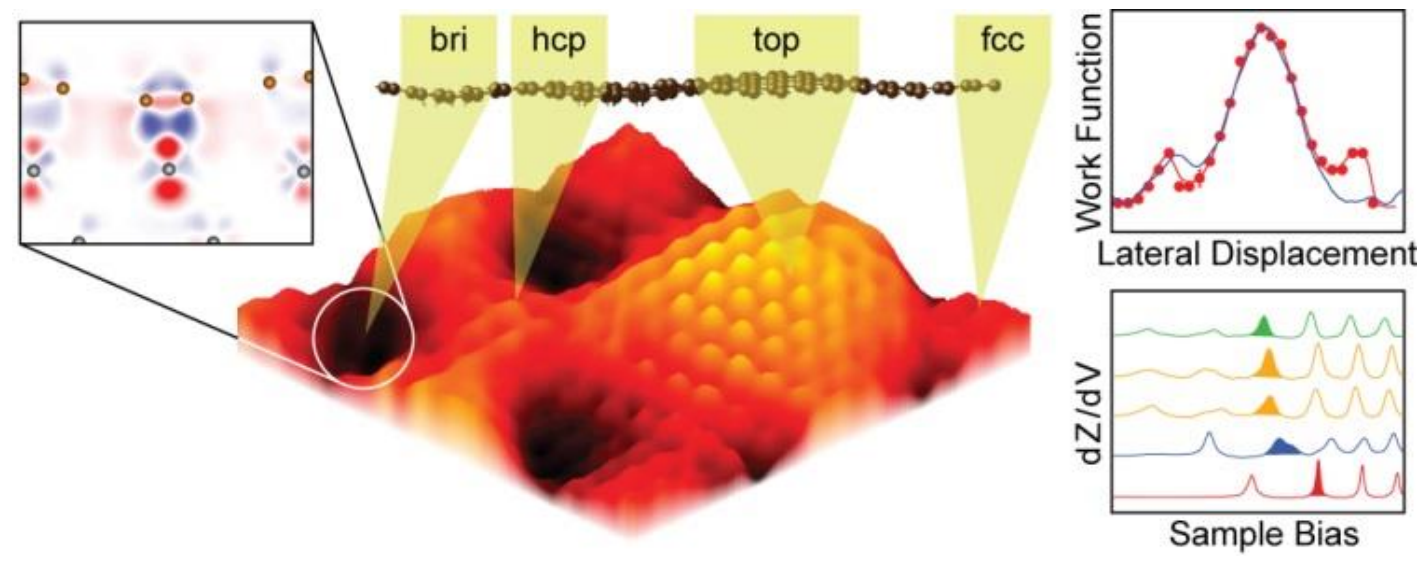

dx.doi.org/10.1021/nn101520k

Received:

July 5,2010

Accepted:

September 24, 2010

Published:

October 1, 2010 
Graphene has attracted considerable interest since researchers at the University of Manchester extracted a single graphene sheet by micromechanical cleavage. ${ }^{1}$ This $\mathrm{sp}^{2}$-bonded carbon network exhibits many unique properties related to the linear dispersion of its low-energy electronic excitations, $^{2}$ and could lead to promising applications for unconventional graphene-based nanoelectronics. Recently, monolayer graphene (MG) was grown on transition metals (TM) by chemical vapor deposition of small hydrocarbons at high temperatures. ${ }^{3-21}$ Hence, MG/TM constitutes an ideal model system for studies pertaining to heterogeneous catalysis. Coke formation (that is the blocking of the active sites of a working catalyst by a carbonaceous overlayer) is a severe problem for the chemical industry. ${ }^{22} \mathrm{MG} / \mathrm{TM}$ also shows potential as a template (nanomesh) for nanotechnology; e.g., polarization-induced surface trapping of laterally organized atoms or molecules was recently demonstrated. ${ }^{11,23}$

The epitaxy of graphene on TMs has been studied by local and nonlocal surface sensitive techniques. Unlike free-standing graphene, MG/TM exhibits various Moiré superstructures, either commensurate $^{6,18,20}$ or incommensurate, 12 depending on the magnitude of the lattice mismatch. In addition, recent scanning tunnelling microscopy ${ }^{4,6,13}$ and photoelectron spectroscopy ${ }^{3}$ studies have indicated that the morphology of graphene correlates with the strength of the orbital hybridization at the interface: graphene grows relatively flat on $5 \mathrm{~d}$ TMs like $\mathrm{Pt}(111)$ and $\operatorname{Ir}(111)$ and is strongly corrugated on 4d TMs like Ru(0001) and $\mathrm{Rh}(111)$. Despite these efforts, very little is known on the coupling between morphology (carbon metal registry) and orbital hybridization (chemical bonding) at the atomic scale. This knowledge is of crucial importance for the applications of graphene, not only in novel electronic devices where charge transfer is related to doping and electron emission properties, ${ }^{24,25}$ but also in catalysis where activation is key to chemical reactivity.

In this work, we investigate the registry of a single layer of graphene with respect to the underlying $\mathrm{Rh}(111)$ substrate at the atomic scale by lowtemperature scanning tunneling microscopy (STM). Our high resolution images show that the variation of the tunneling contrast of graphene on $\mathrm{Rh}(111)$ is much richer than that on other TMs. Notably, we identify a specific local $\mathrm{C}$ - $\mathrm{Rh}$ registry that exhibits a strong contrast minimum in the STM signal unseen for other systems, suggesting that the chemical bonding of graphene with a Rh substrate is different. To investigate this, we performed site-specific resonance tunnelling spectroscopy (RTS) measurements to probe for the modulation of the electronic surface potential (or work function) across the unit cell of the graphene superlattice. By combining microscopy and spectroscopy data, with the support of extended density functional theory (DFT) calculations, the electronic coupling of the various local $\mathrm{C}$ - $\mathrm{Rh}$ registries is examined in detail, whereby we identify experimentally and confirm theoretically the atomic configurations of maximum and minimum chemical bonding to the metal substrate. Our results are at odds with other MG/TM studies and we will explain why this is the case, by providing a discussion of the electronic and structural factors that contribute to the adsorption of MG on TM surfaces.
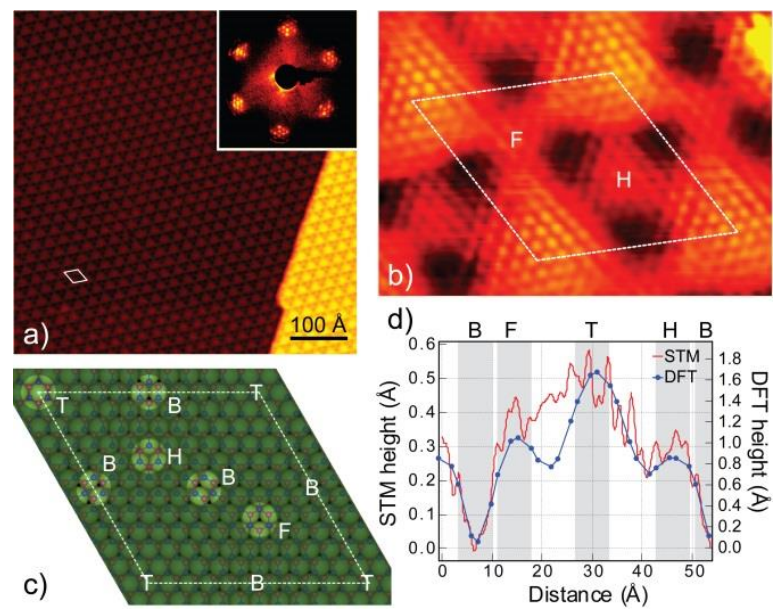

Figure 1: (a) Large-scale STM image of graphene/ $R h(111)$, $60 \times 60 \mathrm{~nm}^{2}$. Inset: LEED pattern of graphene/Rh(111) acquired at $74 \mathrm{eV}$. (b) High-resolution STM image of graphene/Rh(111). The dashed line highlights the unit cell of the Moire superstructure, with side lengths of approximately $2.9 \mathrm{~nm}$. (c) Model of a 12C/11Rh superstructure. The highlighted areas indicate local highsymmetry configurations: $\mathrm{T}=$ ring-top, $\mathrm{B}=$ ring-bridge, $\mathrm{F}=$ ring-fcc, and $\mathrm{H}=$ ring-hcp. Refer to text for a description of the registry labeling. (d) Typical STM line profile (in red) acquired along the main diagonal of a supercell, and height profile (in blue) of the carbon atoms along the main diagonal of the DFT computed 12C/11Rh supercell.

Figure 1a presents a typical STM image of monolayer graphene on a Rh(111) surface. The expected hexagonal Moiré superstructure induced by the lattice mismatch of graphene (2.46 ̊) on $\mathrm{Rh}(111)$ $(2.69 \AA)$ is easily identifiable. The superstructure exhibits a remarkable coherence length, in excess of $100 \mathrm{~nm}$, and is essentially limited by structural defects inherently present on the substrate beneath (such as step edges and other crystalline imperfections). An atomically resolved image of the overlayer is shown in Figure $1 \mathrm{~b}$. The analysis of many similar images shows that the periodicity of the 
superlattice is approximately $29 \AA$. This value corresponds to the expected periodicity of $29.6 \AA$ resulting from a $12 \mathrm{C} / 11 \mathrm{Rh}$ coincidence lattice (where $X / Y$ denotes the size relationship with the underlying substrate: $X$ graphene unit cells accommodated on $Y$ unit cells of the support). In agreement with our topographic observations, LEED patterns (Figure 1a, inset) show hexagonally arranged satellites around the (111) substrate spots, indicative of a superstructure with a periodicity of 12 graphene lattice constants and aligned with the high symmetry directions of the substrate.

Our observations are similar in many aspects to reports of MG on $\left.\operatorname{Ru}(0001),{ }^{4,5} \mathrm{Pd}(111)\right)^{15}$ and $\operatorname{Ir}(111)^{13}$ (these substrates and $\mathrm{Rh}(111)$ share a comparable lattice mismatch of approximately $10 \%$ with graphene), with the exception of one striking difference: a comparison of our high-resolution STM images of MG/Rh(111) (as in Figure 1b) with similar images reported for graphene adsorbed on $\mathrm{Ru}(0001),{ }^{4,5} \operatorname{Ir}(111),{ }^{13}$ and $\mathrm{Pd}(111)^{15}$ reveals that the tunneling contrast over graphene is significantly richer in the case of our Rh substrate (see also the STM line profile shown in Figure 1d). Of particular interest, three regions of pronounced contrast minima are easily distinguished per supercell (their appearance as minima is independent of the STM tip state and the tunneling parameters). These graphitic regions do not show a particularly outstanding characteristic on other TM surfaces. We note that a broad region of maximum contrast stands as the common feature in the STM images of graphene adsorbed on all four substrates.

To discuss the atomic registry within a supercell, we consider the 12C/11TM structure for which a ball model is sketched in Figure 1c. Locally, three C-TM high-symmetry configurations can be distinguished within the supercell, hereafter labeled as ring-top, ring-hollow and ring-bridge, the name referring to the position of the center of a carbon ring with respect to the substrate. ${ }^{26}$ The occurrence of these configurations within a unit cell follows the ratio top: hollow: bridge $=1: 2: 3$. We emphasize that this conclusion remains valid for commensurate relations similar to $12 \mathrm{C} / 11 \mathrm{TM}$ (e.g., a 11C/10TM), hence it is not only applicable to $\mathrm{Rh}(111)$ but also to $\operatorname{Ir}(111)$, $\mathrm{Pd}(111)$, and $\mathrm{Ru}(0001)$ substrates. In light of this ratio, the observed features in our STM images (Figure 1a,b) can straightforwardly be associated with the local high-symmetry configurations. The three low-contrast zones are each identified as ringbridge. The single, large triangular protrusion is assigned to ring-top. To identify the two remaining ring-hollow sites, i.e. -fcc and -hcp, one sees from Figure $1 \mathrm{c}$ that it suffices to distinguish the 3 -fold hollow hcp and fcc substrates sites. This can be easily achieved by imaging the $\mathrm{Rh}(111)$ single-crystal across a step edge with atomic resolution. Note that once this assignment is firmly established, further distinction between the ring-fcc and -hcp registries of graphene becomes trivial: it can be seen from Figure $1 b$ that the three ring-bridge sites define within each supercell a single triangle with darker edges, the center of which corresponds to the ringhcp configuration. As will become apparent later, the unambiguous assignment of the STM topographic features with the local high-symmetry configurations is central to the interpretation and understanding of our spectroscopic measurements and theoretical calculations.

Why is the STM topographic appearance of MG adsorbed on $\mathrm{Rh}(111)$ so different when compared to that of other TM substrates, and in particular to the fairly similar Ru(0001) substrate? This question is of crucial importance for two reasons. First, a variation in the tunnelling contrast necessarily reflects (besides geometrical considerations) a change in local density of electronic states at the Fermi level that depends on the degree of $\mathrm{C}-\mathrm{Rh}$ interaction strength induced by hybridization between the $d$ band of the metal and the $\pi$ states of graphene. Second, the ring-bridge configurations, seen as STM contrast minima on Rh substrates, have so far largely been ignored in previous experimental and theoretical studies. This is unsurprising due to their lack of distinctiveness on other TM substrates. In what follows, we will show that this particular registry corresponds to the local atomic arrangement of strongest chemical bonding between graphene and $\mathrm{Rh}(111)$, in contrast to other MG/TM systems, and we will explain why this is the case by performing registry-specific spectroscopy measurements and extended DFT calculations to probe for work function (WF) differences across the unit cell of graphene/Rh(111). By this approach, we will locally correlate carbon-metal registries and chemical bonding.

Z-V spectra were collected at various locations associated with the high-symmetry configurations discussed above, and on the clean Rh(111) surface for comparison. Figure 2 shows the corresponding $\mathrm{dZ} / \mathrm{dV}$ spectra obtained after numerical differentiation of the Z-V data. The spectra exhibit a complex structure, but upon close inspection, two series of resonances can be identified: A first series, labeled $m$, occurs in the low voltage range (2-5 V) and is distinguished by broad peaks of low intensity. A second series, labeled $n$, occurs in the higher voltage range $(4-10 \mathrm{~V})$ and is characterized by sharper, intense peaks. The $n$ series is induced by the 
well-established resonance tunnelling mechanism (in field emission) between the STM probe and the substrate. $^{27,28}$ Importantly, Rh(111) exhibits only the $\mathrm{n}$ series (with a first resonance at an energy of 5.84 $\mathrm{eV}$, well above its WF of $5.6 \mathrm{eV}^{29}$ ). The $m$ series is induced by resonance states located between graphene and the substrate. $^{30}$

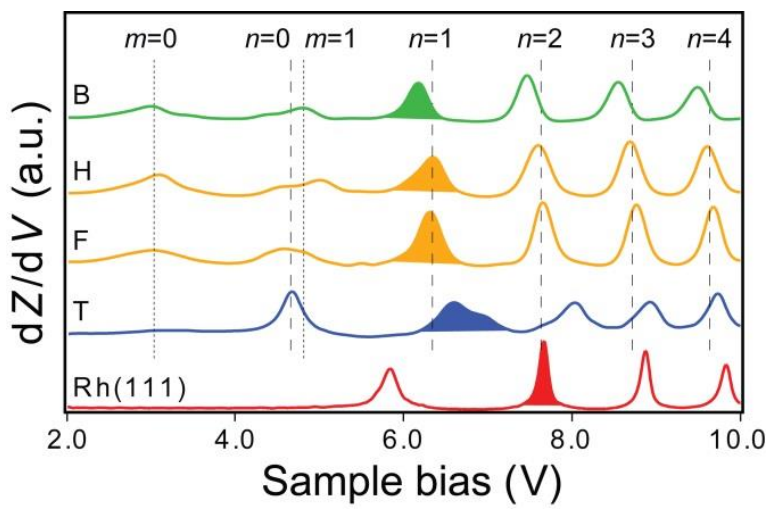

Figure 2: $d z / d V$ spectra acquired at different locations on graphene/Rh(111) and on $\mathrm{Rh}(111)$ for reference. Tunneling current of $1.0 \mathrm{nA}$. The $n=1$ resonance is highlighted.

We first discuss the $\mathrm{n}$ series. It was recently reported by Lin et al. ${ }^{31}$ that the energy shift of the second field emission resonance $(n=1)$ is, to a very good approximation, equal to the WF difference between two sites on a surface (every spectrum in Figure 2 is an average of five measurements; we estimate the error in energy to be $\pm 10 \mathrm{meV}$ ). Lin and co-workers explain that the $n=1$ peak is less affected by the image potential of the surface and, in our case, is also energetically well separated from the $m$ resonance series (while the $n=0$ peak overlaps with the $m=1$ peak). Figure 2 reveals that ring-top and ring-bridge sites possess a different resonance spectrum, whereas the two ring-hollow sites give similar spectra, with very small energy shifts. The ring-top site is observed to have a much higher WF than other sites, and the ring-bridge the lowest WF. From comparison with the $\mathrm{dZ} / \mathrm{dV}$ spectrum of $\mathrm{Rh}(111)$ and with reference to its WF $\left(5.6 \mathrm{eV}^{29}\right)$, we can deduce absolute values for the local WF of graphene: ${ }^{31} 4.50 \mathrm{eV}$ for the ring-top registry, and within the range 4.25 to $4.30 \mathrm{eV}$ for the other sites. The WF of the ring-top registry is remarkably similar to the expected value for freestanding graphene (4.5 $\mathrm{eV}),^{32,33}$ indicating a locally weak C-Rh interaction. In contrast, the other sites are seen to interact substantially with the substrate since a variation in their WF signifies an increased hybridization with the $\mathrm{Rh} 4 \mathrm{~d}$ orbitals.

Figure 3a presents a color-coded series of $d Z / d V$ spectra recorded along the line indicated in the STM image of Figure $3 \mathrm{~b}$ encompassing all local $\mathrm{C}-\mathrm{Rh}$ registries. The continuous WF change across the entire supercell (as deduced from the energy shift of the $n=1$ oscillation $^{31}$ ) is reported in Figure $3 \mathrm{c}$. In line with our previous observations, the WF reaches a maximum at the center of ring-top sites and a minimum at ring-bridge sites, with an overall variation of $220 \mathrm{meV}$. In addition, two local WF minima are identified on each side of ring-top. These do not coincide with the centers of the ring-fcc or the ring-hcp sites, offset by about $3 \AA$ along the main diagonal of the supercell. On the basis of this fact and the ball model in Figure 1c, these two sites of local WF minima (referred to as "ring-asymmetric" sites) are seen to adopt a structure in which one carbon atom resides nearly on top of a $\mathrm{Rh}$ atom while the other resides above a bridge site between two Rh atoms, as shown in Figure $3 d$.

a)

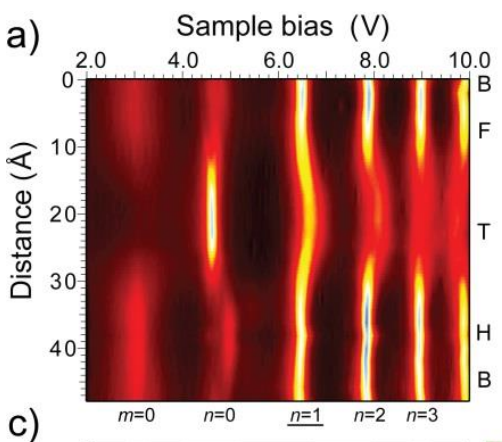

b)
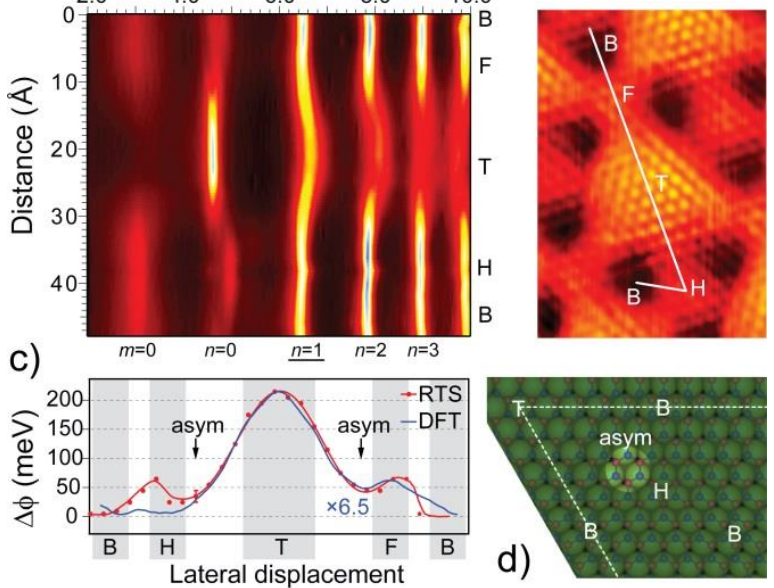

Figure 3: (a) Series of $d Z / d V$ spectra $(1.0 \mathrm{nA})$ recorded along the line indicated in the STM image in panel b. (b) STM image, $5 \mathrm{~nm} \times 3.5 \mathrm{~nm}, 0.5 \mathrm{nA}$, and $0.4 \mathrm{~V}$. (c) Work function versus position as deduced from the $n=1$ peak in panel a. The red dots are the experimental data points and the blue curve is extracted from DFT calculations. (d) Atomic model for the ring-asymmetric registry of the local minimum work function.

We now discuss the $m$ series of resonance peaks. This series is not present in the spectrum of clean $\mathrm{Rh}$ (111) (Figure 2). We hence interpret these peaks as resulting from resonance states within the buried interface defined by the scattering planes on each side of the graphene/Rh(111) boundary. Similar oscillations at a low bias have been previously observed by Kubby et al. in the case of first-layer subsurface vacancies in $\mathrm{Si}(111) .^{30}$ The intensity and width of such resonances are related to the lifetime of the excitation, ${ }^{34}$ that is, to the probability for tunneling out of the well region through either of the two enclosing barriers. As shown in Figures 2 and $3 a$ (see the $m=0$ peak), these resonances are almost 
absent in the large region of the ring-top configuration, but are quite intense in other zones. Consequently, the resonances in the ring-top configuration are necessarily shorter lived than those in other regions of the supercell. We can rationalize this observation by reasonably assuming that the scattering strength of the barriers compares with the bonding strength between graphene and the $\mathrm{Rh}(111)$ substrate, as discussed by Zhang et al., ${ }^{35}$ and will therefore be weaker for ring-top sites in agreement with our previous discussions.

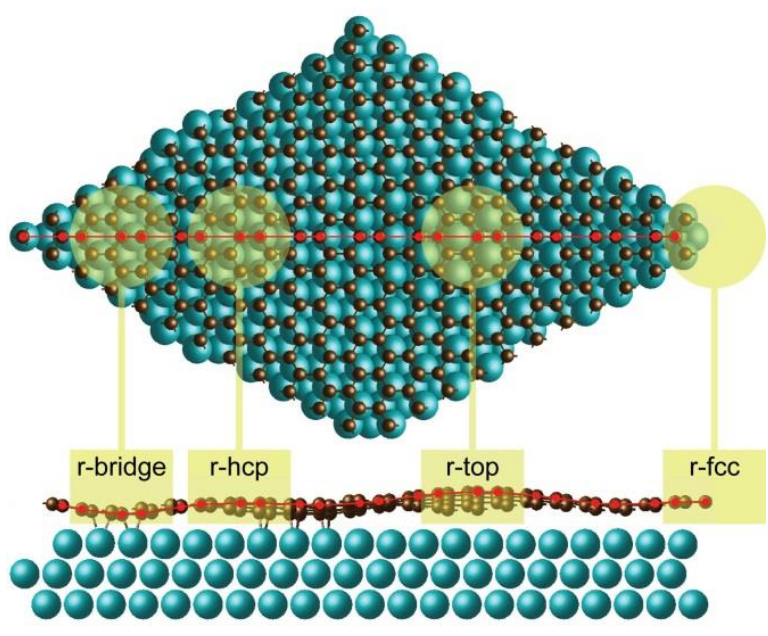

Figure 4: DFT relaxed structure (top and side views) of the $12 \mathrm{C} / 11 \mathrm{Rh}(111)$ surface. The atomic configurations of the supercell corresponding to the local high-symmetry registries are highlighted with the yellow-shaded areas. The red circles and lines indicate the positions of the carbon atoms along the long diagonal of the supercell.

To substantiate our experimental findings and to obtain deeper insights into the chemical bonding at the interface, we performed extended DFT calculations on a $12 \mathrm{C} / 11 \mathrm{Rh}$ supercell. Figure 4 shows the relaxed configuration (top and side views). The graphitic overlayer displays a strong height modulation with respect to the first $\mathrm{Rh}(111)$ layer, the latter being slightly buckled by $0.1 \AA$ in antiphase with the MG corrugation. ${ }^{7,8}$ As expected, the ringtop registry lies highest, with a C-Rh distance of 3.8 $\AA$. The ring-fcc and -hcp registries are located at 3.1 and $2.9 \AA$ from the Rh surface, respectively. The ringbridge registry lies lowest at a distance of $2.2 \AA$. This is clearly different from extended DFT calculations performed on $\mathrm{Ru}(0001)^{36,37}$ and $\operatorname{Ir}(111) .{ }^{11}$ First, the lowest lying registries were identified as ring-hcp in all cases. These studies do not refer to the ringbridge registry specifically, but we understand that this configuration adopts a height slightly larger than, if not equal to ring-hcp. Besides a pronounced hump at ring-top sites, the graphitic overlayers on Ir and $\mathrm{Ru}$ are predicted to be practically flat and featureless. Second, we measure from our computational data a buckling for graphene on $\mathrm{Rh}(111)$ of $1.6 \AA$, judged against $1.5 \AA^{37}$ and $1.7 \AA^{36}$ for $\mathrm{Ru}(0001)$ and $0.3 \AA$ for $\operatorname{Ir}(111) .{ }^{11}$ Hence, the theoretical corrugations of $M G$ adsorbed on $\mathrm{Ru}, \mathrm{Rh}$, and $I r$ indicate that the interaction strength follows the sequence $\mathrm{Ru} \sim \mathrm{Rh}>\mathrm{Ir}$. A surprising aspect, however, arises when comparing the C-TM distances at the various bonding registries for the $\mathrm{Ru}$ and $\mathrm{Rh}$ substrates. Although the ring-bridge sites are similar (lying in both cases at $2.2 \AA$ above the surface), the ring-hollow configurations differ by about $1 \AA$. This difference is significant enough to suggest that the $C$ TM bonding strength at these sites is considerably reduced on $\mathrm{Rh}$ (compare the distances of about 2.2 $\AA$ on $\mathrm{Ru}^{36,37}$ and 2.9-3.1 $\AA$ on $\mathrm{Rh}$ ). Importantly, we compare in Figure $1 \mathrm{~d}$ the experimental and theoretical height profiles for the $\mathrm{MG} / \mathrm{Rh}(111)$ system. Keeping in mind that the overall corrugation amplitude as measured by STM is necessarily dependent on the tunneling voltage and STM tip state, the relative agreement with our theoretical predictions is remarkable. Only the heights of the $C$ atoms in the ring-fcc configuration are calculated to be slightly lower by theory than is observed experimentally.

To examine the origin of the differences in the local bonding, we computed the electron density difference map for the $12 \mathrm{C} / 11 \mathrm{Rh}$ supercell, shown in Figure $5 \mathrm{a}$. The top panel depicts an iso-value surface (as seen from above) of 0.02 electrons $/ \AA^{3}$ in the difference between the electron density of the Rh-C system and the sum of its constituents calculated at the geometry of the combined system. Blue indicates higher electron density (i.e., negative charge) on the combined system, red shows a reduction in electron density. We observe that the chemical bonding strength varies significantly across the supercell. The local registries associated with ring-top do not show any charge redistribution between the adsorbed graphitic overlayer and the substrate, indicative of an absence of hybridization. A bonding character to the $\mathrm{C}-\mathrm{Rh}$ interaction can, however, be inferred for the other parts of the supercell, with an adsorption strength following the sequence ring-fcc $<$ ring-hcp $<$ ring-bridge.

The bottom panel of Figure 5a displays a cut through the electron density change along the main diagonal of the supercell. An accumulation of charge density at the interface between graphene and $\mathrm{Rh}$ is evident at the bonding registries only. Focusing first on the ring-hcp configuration (ring-fcc is similar), we see that chemical bonding to the Rh substrate occurs mainly through the carbon atoms on atop sites, as a result of hybridization between the $C\left(\pi / \pi^{*}\right)$ and $\mathrm{Rh}(\mathrm{d})$ bands. The same conclusions were drawn for 


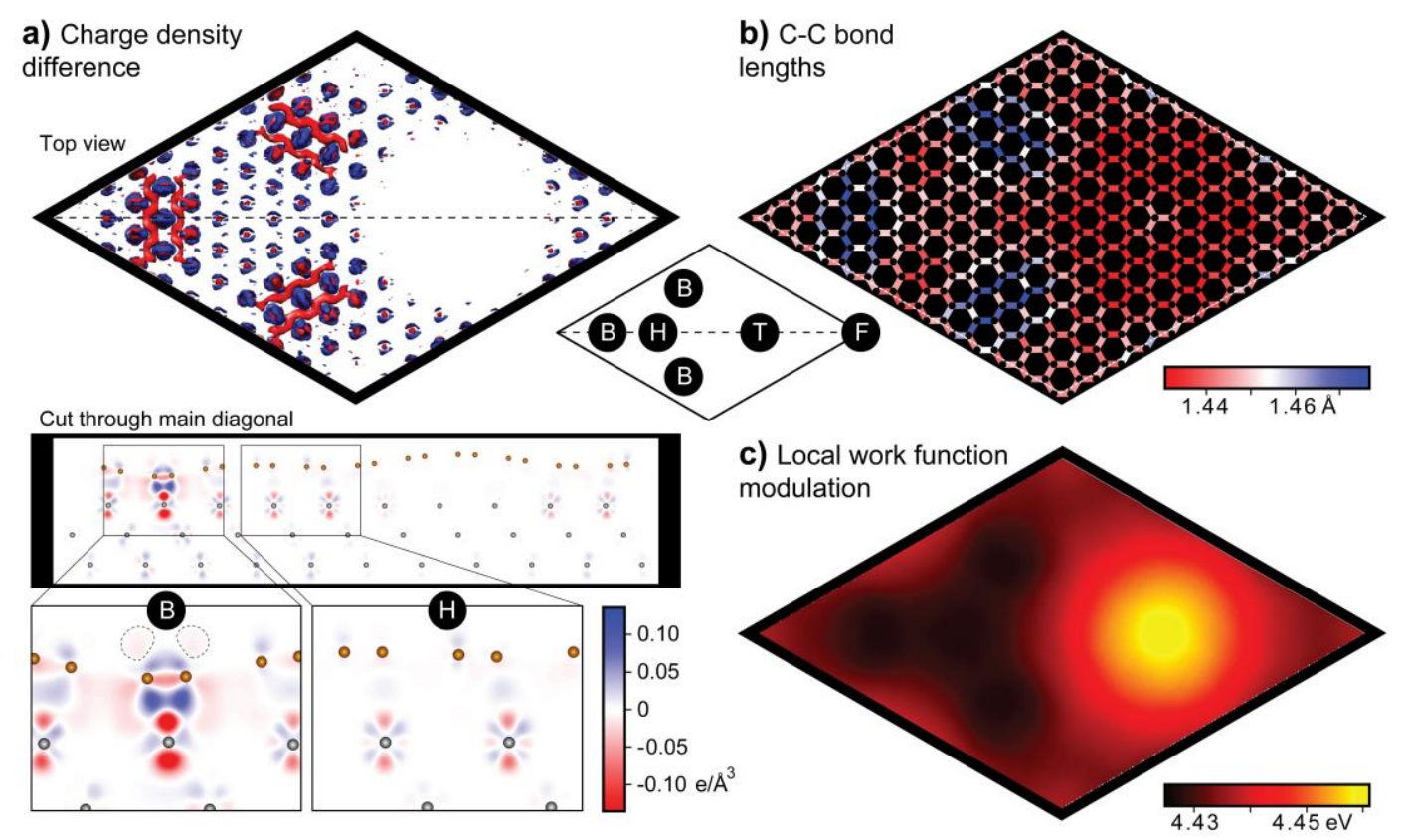

Figure 5: (a) Computed adsorption-induced charge density difference map for a $12 \mathrm{C} / 11 \mathrm{Rh}$ supercell of graphene/Rh(111). Blue and red indicate an increase and a decrease, respectively, of electron density. The top panel depicts an electronic iso-surface at 0.02 electrons $/ \AA^{3}$. The bottom panel shows a cut through the charge density along the main diagonal of the supercell, alongside two higher magnification plots highlighting the density change locally associated with the ring-bridge (left) and ring-hcp (right) configurations. The area delimited by the dashed line highlights a decrease in the overspill of the surface charge density into the vacuum at the ring-bridge site. (b) Colorcoded representation of the $\mathrm{C}-\mathrm{C}$ bond lengths within a supercell. (c) Calculated work function variation over a supercell. Yellow indicates a higher work function, and black a lower. The small supercell in the middle serves as a guide to help with the location of the high-symmetry epitaxial registries ( $T$ = ring-top, $H=$ ring-hcp, $F=$ ring-fcc, and $B=$ ring-bridge).

ring-hollow sites interacting with $\mathrm{Ru}$ and $\mathrm{Ir}$ substrates, ${ }^{11,36,37}$ involving a dominating backdonation with a net negative charge acquired by the graphene layer. Even so, a comparison with similar DFT data for $\mathrm{MG} / \mathrm{Ru}^{36,37}$ highlights that the interaction at these sites is significantly reduced on the Rh substrate.

The electronic intermixing between the graphitic $\pi / \pi^{*}$ system and the metal $d$ bands is, on the other hand, markedly different at the ring-bridge sites. First, it involves two carbon atoms symmetrically and laterally displaced from the atop position. Second, the charge redistribution is significantly enhanced in comparison to the ring-hollow sites. These two observations suggest that a different contribution of the various $\mathrm{Rh}(\mathrm{d})$ orbitals to the bonding with carbon must take place at ring-bridge sites. We examine this in Figure 6 by comparing the orbital decomposed local density of states (LDOS) for C and Rh atoms representative of the various local high-symmetry configurations (ring-top, -hcp, and -bridge). The $\operatorname{Rh}\left(d_{z 2}\right)$ and $C\left(p_{z}\right)$ orbitals show no sign of hybridization at ring-top sites. At ring-hollow sites, one of the two $C\left(p_{z}\right)$ orbitals (corresponding to the atop C) hybridizes with $\mathrm{Rh}\left(\mathrm{d}_{\mathrm{z} 2}\right)$. This is evident from the appearance of shared density around the Fermi level (defined at $0 \mathrm{eV}$ ), and is in line with theoretical predictions made at ring-hcp sites for $\mathrm{MG} / \mathrm{Ru}(0001) .{ }^{36}$ Importantly, we note that $\mathrm{C}\left(\mathrm{p}_{z}\right)$ hardly interacts with the two $R h\left(d_{z x}\right)$ and $R h\left(d_{y z}\right)$ orbitals, as expected from the local adsorption geometry at these sites. However, Figure 6 clearly indicates that the $p_{z}$ orbitals of both $C$ atoms in the ring-bridge configuration couple strongly to the $\mathrm{Rh}(\mathrm{dz2})$ orbital as well as to $\mathrm{Rh}\left(\mathrm{d}_{\mathrm{zx}}\right)$ and $\mathrm{Rh}\left(\mathrm{d}_{\mathrm{yz}}\right)$. A similar donation/back-donation binding mechanism applies here, whereby the C-Rh bonds are not only strengthened, but the $\mathrm{sp}^{2} \mathrm{C}-\mathrm{C}$ bonds are weakened. Our DFT calculations indicate that the overall tensile stress in the graphene sheet resulting from its $12 \mathrm{C} / 11 \mathrm{Rh}$ epitaxy amounts to $1 \%$ with an average C$\mathrm{C}$ bond length of $1.439 \AA$ (compared to $1.424 \AA$ for freestanding graphene). Figure $5 b$ shows the distribution of bond lengths within the computed supercell. While the large majority of the bonds retain the average length (the red bonds in Figure 5b), the carbon atoms in the ring-bridge configurations have two of their three bonds increased by a further $3 \%$, thus becoming weaker. 
Interestingly, it can be seen from the ball-model shown in Figure 1c, and in light of the bond lengths depicted in Figure 5b, that the resulting uniaxial lattice stretch near ring-bridge configurations brings four carbon atoms (out of the six-membered benzene unit) closer to atop positions, thereby increasing head-to-head overlap between the $C\left(p_{z}\right)$ and $\mathrm{Rh}\left(\mathrm{d}_{\mathrm{z} 2}\right)$ orbitals. Hence, the significantly enhanced chemical bonding of ring-bridge sites to the substrate arises as a natural consequence of the substantial $C\left(\pi / \pi^{*}\right)$ coupling with the $\operatorname{Rh}\left(d_{z 2}\right), \operatorname{Rh}\left(d_{z x}\right)$, and $\mathrm{Rh}\left(\mathrm{d}_{\mathrm{yz}}\right)$ orbitals leading to a local weakening of the $\mathrm{sp}^{2}$ carbon network.

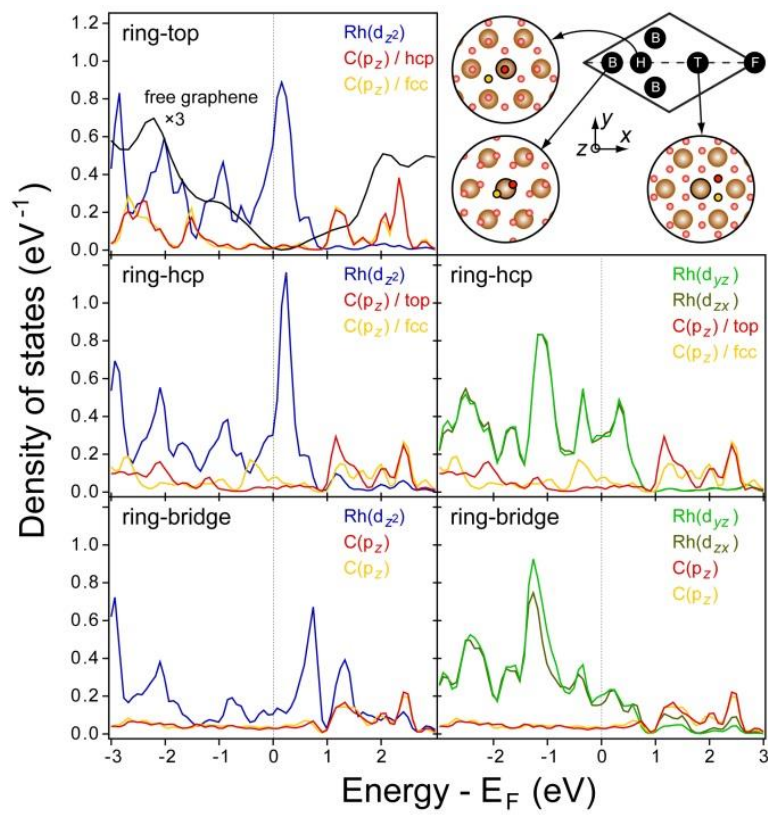

Figure 6: Orbital decomposed local density of states (LDOS) for $\mathrm{C}$ and $\mathrm{Rh}$ atoms in the various local highsymmetry configurations (ring-top, -hcp, and -bridge). The LDOS for the adsorbed MG/Rh(111) system is represented by the colored traces. The atoms, for which the LDOS is presented, are highlighted in the supercell shown on the top-right corner, alongside the $x, y$, and $z$ coordinate system used. The computed total DOS of free-standing graphene is appended for reference (black trace in the ring-top panel).

Our DFT calculations allow us to predict (and ultimately verify) how the WF of graphene will alter upon adsorption. Since no electron redistribution is associated with the ring-top configurations, we expect the local WF at these sites to be equal to the WF of freestanding graphene, i.e. $4.5 \mathrm{eV}^{32,33}$ In contrast, a net negative charge is transferred to the $\mathrm{MG} / \mathrm{Rh}$ interface for all other configurations. Adsorbate-induced reduction of the WF can easily be understood as the result of a net charge transfer from the adsorbate to the substrate, opposite to that indicated by our experimental data. Such abnormal WF change, however, has been previously observed in surface adsorption studies ${ }^{38}$ and was explained to arise from a dipolar counteraction due to charge redistribution within the adsorbates. The charge redistribution on the carbon atoms at ringbridge sites is indeed accompanied by a decrease in the overspill of the surface charge density into the vacuum (see the area enclosed by the dashed-line in the bottom panel of Figure 5a). We can hence expect the ring-bridge configurations to exhibit the lowest WF and the ring-hollow sites to have an intermediate WF.

To extract from our DFT data the WF variation across the $12 \mathrm{C} / 11 \mathrm{Rh}$ supercell, we determined the local potential in the vacuum by choosing the plane in the middle between the Rh slabs and subtract the Fermi energy sampled over a fine surface mesh to account for all local C-Rh registries. To allow for a direct comparison with our spectroscopy measurements, the computed WF is further smoothed with use of a two-dimensional Gaussian filter with a width of 0.75 $\mathrm{nm}$ (to simulate the STM tip convolution and to account for the inherent lateral resolution of RTS measurements as described in ref 27). The result is shown in Figure $5 c$. In line with our predictions, we observe that the WF is highest for ring-top sites (calculated at $4.461 \mathrm{eV}$ ), and lowest for ring-bridge sites (calculated at $4.427 \mathrm{eV}$ ), yielding a theoretical WF modulation of $34 \mathrm{meV}$ across the supercell, roughly a factor of 6 smaller than the $220 \mathrm{meV}$ experimentally measured. Nonetheless, a profile of the computed WF along the main diagonal of the supercell can now be directly compared to our experimental results. As shown in Figure $3 c$, the excellent qualitative agreement is obvious. In particular, it can be seen that the relative WF variation at the different high-symmetry sites is remarkably reproduced with the exception of the ring-hcp configuration. The experimental identification of the ring-asymmetric sites associated with local minima (or more precisely with saddle points as seen from the 2D representation in Figure 5c) in the WF is also confirmed. The theoretical 6fold underestimate of the WF modulation and the deviation at ring-hcp sites are likely to arise from the fact that three $\mathrm{Rh}$ layers are not sufficient to describe the electronic structure of the bulk metal. We have not considered testing for this, given the computationally very demanding nature of the $12 \mathrm{C} / 11 \mathrm{Rh}$ supercell simulation. Note, however, that N'Diaye and co-workers report that the corrugation of $M G / \operatorname{Ir}(111)$ and its binding energy are insensitive to the addition of a fourth substrate layer. ${ }^{11}$ The excellent qualitative agreement reached here, nevertheless, indicates that our calculations are 
accurate enough to describe the qualitative trend in the surface electronic potential.

In essence, our combined microscopy, spectroscopy, and theoretical data identify ring-top and ring-bridge sites as the local configurations of minimum and maximum chemical bonding to the substrate, respectively, with $\mathrm{C}-\mathrm{Rh}$ bond lengths following the sequence: ring-bridge $<-\mathrm{hcp} \sim-\mathrm{fcc}<-$-top. Since there exists a qualitative dependence between the strength of the chemical interaction and the degree of geometric corrugation at the MG/TM interface, ${ }^{3}$ our data provide evidence that $\mathrm{MG} / \mathrm{Rh}(111)$ is corrugated at the atomic scale, with the ring-top sites protruding from the surface and the ring-bridge sites lying the closest. Interestingly, our observations indicate that the tunnelling contrast acquired in the low bias regime mirrors on a relative scale the topography of the MG/Rh(111) surfaces. If so, similar conclusions could (and indeed seem to) apply to MG adsorbed on $\mathrm{Ru}(0001)$ and $\operatorname{Ir}(111)$, although one needs to be vigilant for possible STM effects (e.g., tunneling parameter-dependent contrast reversal as reported for $M G / R u(0001)^{4}$ and $M G / \operatorname{Ir}(111)^{12}$ ). Considering that the STM contrast is a convolution of not only geometric but also electronic contributions, such an assessment is a priori ambiguous without our present investigation, and has been the subject of an intense dispute for MG/TMs. ${ }^{4,5}$

Although many of our conclusions reached for $\mathrm{Rh}(111)$ hold true for other TM substrates, the observed trend in the local adsorption strength (ringhollow < ring-bridge) is at odds with the consensus that emerges from the literature regarding the most favourable local adsorption registry for MG/TM, reported experimentally and/or theoretically to be ring-hcp for the close-packed surfaces of $\mathrm{Ru}^{4,36,37}$ $\mathrm{Pd},{ }^{15} \mathrm{Pt},{ }^{39}$ and $\mathrm{Ir}^{11-13}$ In addition to these $4 \mathrm{~d}$ and $5 \mathrm{~d}$ metals, which exhibit a comparable $10 \%$ lattice mismatch with graphene, the surfaces of $\mathrm{Co}(0001)^{17,18}$ and $\mathrm{Ni}(111),^{19-21,40}$ both $3 \mathrm{~d}$ metals with a lattice mismatch reduced to about $1 \%$, have been experimentally shown to adsorb graphene in a $(1 \times 1)$ epitaxy favoring the ring-hollow geometry. As previously mentioned, ring-bridge sites have escaped the attention of most studies to date. Swart et al. report that this registry is significantly less stable on fcc-Co(111). ${ }^{26}$ It is worth mentioning that one theoretical study reports that the ring-bridge structure is favored over ring-hcp on $\mathrm{Ni}(111)^{41}$ but this conclusion can be disputed on the grounds of recent experimental findings. ${ }^{20,21,40}$

Furthermore, both Ru and Rh (4d metals) are more active than Ir (a $5 \mathrm{~d}$ metal). One naively expects that MG should bind similarly to $\mathrm{Rh}$ and $\mathrm{Ru}$, but significantly less to Ir. However, the adsorption energies for MG/TM, averaged over extended supercells, are $40 \mathrm{meV}$ per carbon atom over $\mathrm{Ru}(0001){ }^{37} 7.8 \mathrm{meV} / \mathrm{C}$ over Rh(111) (from our DFT data), and $2 \mathrm{meV} / \mathrm{C}$ over $\operatorname{Ir}(111) .{ }^{11}$ Additionally, one expects that the overall physical characteristics of the MG/Rh(111) interface should be comparable to those of the MG/Ru(0001) system, with only slightly larger C-TM bond distances for the former, and certainly not an inversion in relative bonding strength between ring-hcp and ring-bridge sites. The experimentally and/or theoretically deduced MG-TM distances reported in the literature (for the bonding configurations ring-bridge and ring-hollow) are about 2.0-2.2 $\AA$ for the close-packed surfaces of both $3 \mathrm{~d}$ and $4 \mathrm{~d}$ TM substrates $\left(\mathrm{Ni}^{19}{ }^{19} \mathrm{Co},{ }^{17} \mathrm{Ru}^{7,8} \mathrm{Pd}^{42}\right.$ ), and 3.3-3.8 $\AA$ for $5 \mathrm{~d}$ metals $\left(\mathrm{Pt},{ }^{39} \mathrm{Ir}^{11}\right)$. The case of $\mathrm{Rh}(111)$ is hence very intriguing, in that it follows a "4d trend" with its two neighbors Ru and Pd with respect to the ring-bridge configuration, whereas its two ring-hollow configurations exhibit more of a " $5 d$ metal" attribute.

To provide a qualitative explanation to the differences in the bonding of graphene to the various group VIIIB TMs, we need to separate two contributing factors to the binding energy: electronic (energy and symmetry of the states involved) and structural (mismatch and structure of the lattice). To a first approximation, the electronic factor can be described by the $d$ band model. ${ }^{43}$ However, this model is inappropriate in the present case because it neglects to include (1) structural considerations such as the lattice mismatch at the MG/TM interface and the crystal structure of the TM support, e.g. hcp vs $\mathrm{fcc}$, and (2) electronic effects arising from an appropriate many-electron description, including exchange and correlation energies. It is very wellknown that these two neglected aspects are of fundamental importance for a coherent understanding of epitaxial graphene and its unique properties related to the linear dispersion of its lowenergy electronic excitations. Due to the lattice mismatch, graphene is forced to adsorb at different sites whereby variations in the electronic structure induce differences in chemical bonding. Hence, the local registries of maximum and minimum chemical bonding, and the magnitude of the buckling, relate to differences in the interaction strength between different sites.

We finally note, with reference to studies of MG adsorbed on 3d, 4d, and 5d TM surfaces that allow for tangible comparisons to be made (similar STM and DFT-GGA approaches), that the overall agreement between experimental and theoretical data based on DFT is rather good, thereby yielding a 
reliable description of the MG/TM interface, provided the correct structural features are considered, e.g. the extended supercell to account for the lattice mismatch. We emphasize here that slight computational differences known to arise from the use of LDA versus GGA, especially in relation to bond distances, do not undermine the present discussion.

In summary, we have explored by means of highresolution STM, site-specific RTS, and extended DFT calculations the local adsorption registry and electronic coupling between monolayer graphene and a $\mathrm{Rh}(111)$ substrate. We measured a work function modulation of about $0.22 \mathrm{eV}$, which is significant in light of the spatial extent over which it occurs (approximately $3 \mathrm{~nm}$, the size of a 12C/11Rh supercell). We have confirmed that epitaxial graphene is geometrically buckled when adsorbed on $\mathrm{Rh}(111)$. Furthermore, we identified, with both experiment and theory, the local registry of maximum chemical bonding of graphene to the $\mathrm{Rh}$ (111) substrate. We finally discussed the various factors involved in the bonding strength of MG on various TM surfaces, thereby rationalizing our data for the $\mathrm{Rh}(111)$ substrate.

\section{METHODS}

Our experiments were performed in an ultrahighvacuum chamber with a base pressure below $1 \times 10^{-10}$ mbar. The $\mathrm{Rh}(111)$ crystal was cleaned by repeated cycles of $\mathrm{Ar}^{+}$sputtering and annealing in oxygen $\left(3 \times 10^{-7} \mathrm{mbar}\right)$ at $1100 \mathrm{~K}$, followed by a final flashanneal in vacuum to $1200 \mathrm{~K}$. Exposure to ethylene at $1100 \mathrm{~K}\left(2 \times 10^{-7} \mathrm{mbar}, 3 \mathrm{~min}\right)$ results in the formation of a single graphene layer on $\mathrm{Rh}(111)$, as reported for other TM surfaces. ${ }^{4,13}$ STM and RTS measurements were performed at liquid nitrogen and helium temperatures, respectively.

Periodic DFT calculations were carried out with use of the VASP code ${ }^{44-46}$ with the gradient corrected PW91 functional ${ }^{47}$ and projector augmented wave (PAW) potentials. ${ }^{48}$ Our simulations employed a plane wave basis with a kinetic energy cutoff of 300 $\mathrm{eV}$ for geometry optimization, and $400 \mathrm{eV}$ for subsequent charge density differences and density of states calculations. Sampling of the Brillouin zone was made by using only the $\Gamma$-point, which can be justified through the size of the unit cell of the graphitic superstructure (29.9 $\AA$ in the plane of the surface). To model the geometry and electronic structure of the MG/Rh(111) system, we constructed a unit cell with a 12C/11Rh coincidence lattice. This reflects our experimental observations as described above. The simulated system consists of a graphene sheet adsorbed on three rhodium layers. The unit cell of the graphitic superstructure contains 288 carbon and 363 rhodium atoms. The lower two Rh layers were frozen at the bulk geometry, while the uppermost layer of metal atoms and the carbon layer were optimized with an energy tolerance set at $0.02 \mathrm{eV}$. Surface dipole correction in the direction orthogonal to the surface was applied. The size of the unit cell orthogonal to the surface was set to $33.31 \AA$. The optimized lattice constants for graphene and $\mathrm{Rh}$ are 2.466 and $2.718 \AA$, respectively, in good agreement with experimental values $(2.46$ and $2.69 \AA$, respectively). Our DFT calculations follow the procedures reported by other groups for MG on $\mathrm{fcc} / \mathrm{Co}(111),{ }^{26} \mathrm{Ru}(0001),{ }^{36,37}$ and $\operatorname{Ir}(111),{ }^{11}$ allowing for meaningful comparisons to be drawn.

\section{ACKNOWLEDGEMENTS}

We acknowledge financial support from the Scottish Funding Council, through EaStCHEM, and the SRDG grant HR07003. Computations were carried out on the EaStCHEM Research Computing Facility.

\section{REFERENCES}

1. Novoselov, K. S.; Geim, A. K.; Morozov, S. V.; Jiang, D.; Zhang, Y.; Dubonos, S. V.; Grigorieva, I. V.; Firsov, A. A. Science 2004, 306, 666-669.

2. Novoselov, K. S.; Geim, A. K.; Morozov, S. V.; Jiang, D.; Katsnelson, M. I.; Grigorieva, I. V.; Dubonos, S. V.; Firsov, A. A. Nature 2005, 438, 197-200.

3. Preobrajenski, A. B.; Ng, M. L.; Vinogradov, A. S.; Mårtensson, N. Phys. Rev. B 2008, 78, 073401.

4. Vazquez de Parga, A. L.; Calleja, F.; Borca, B.; Passeggi, M. C. G. J.; Hinarejos, J. J.; Guinea, F.; Miranda, R. Phys. Rev. Lett. 2008, 100, 056807.

5. Marchini, S.; Günther, S.; Wintterlin, J. Phys. Rev. B 2007, 76, 075429.

6. Martoccia, D.; Willmott, P. R.; Brugger, T.; Björck, M.; Günther, S.; Schlepütz, C. M.; Cervellino, A.; Pauli, S. A.; Patterson, B. D.; Marchini, S.; Wintterlin, J.; Moritz, W.; Greber, T. Phys. Rev. Lett. 2008, 101, 126102.

7. Martoccia, D.; Björck, M.; Schlepütz, C. M.; Brugger, T.; Pauli, S. A.; Patterson, B. D.; Greber, T.; Willmott, P. R. New J. Phys. 2010, 12, 043028.

8. Moritz, W.; Wang, B.; Bocquet, M. L.; Brugger, T.; Greber, T.; Wintterlin, J.; Günther, S. Phys. Rev. Lett. 2010, 104, 136102.

9. Wu, M.-C.; Xu, Q.; Goodman, D. W. J. Phys. Chem. 1994, 98, 5104-5110.

10. Sicot, M.; Bouvron, S.; Zander, O.; Rüdiger, U.; Dedkov, Y. S.; Fonin, M. Appl. Phys. Lett. 2010, 96, 093115.

11. N'Diaye, A. T.; Bliekamp, S.; Feibelman, P. J.; Michely, T. Phys. Rev. Lett. 2006, 97, 215501.

12. N'Diaye, A. T.; Coraux, J.; Plasa, T. N.; Busse, C.; Michely, T. New J. Phys. 2008, 10, 043033.

13. Coraux, J.; N'Diaye, A. T.; Busse, C.; Michely, T. Nano Lett. 2008, 8, 565-570.

14. Pletikosic, I.; Kralj, M.; Pervan, P.; Brako, R.; Coraux, J.; N’Diaye, A. T.; Busse, C.; Michely, T. Phys. Rev. Lett. 2009, 
102, 056808.

15. Kwon, S.-Y.; Ciobanu, C. V.; Petrova, V.; Shenoy, V. B.; Bareno, J.; Gambin, V.; Petrov, I.; Kodambaka, S. Nano Lett. 2009, 9, 3985-3990.

16. Nakagoe, O.; Takagi, N.; Matsumoto, Y. Surf. Sci. 2002, 514, 414-419.

17. Eom, D.; Prezzi, D.; Rim, K. T.; Zhou, H.; Lefenfeld, M.; Xiao, S.; Nuckolls, C.; Hybertsen, M. S.; Heinz, T. F.; Flynn, G. W. Nano Lett. 2009, 9, 2844-2848.

18. Varykhalov, A.; Rader, O. Phys. Rev. B 2009, 80, 035437.

19. Gamo, Y.; Nagashima, A.; Wakabayashi, M.; Terai, M.; Oshima, C. Surf. Sci. 1997, 374, 61-64.

20. Dedkov, Y. S.; Fonin, M.; Rüdiger, U.; Laubschat, C. Phys. Rev. Lett. 2008, 100, 107602.

21. Varykhalov, A.; Sanchez-Barriga, J.; Shikin, A. M.; Biswas, C.; Vescovo, E.; Rybkin, A.; Marchenko, D.; Rader, O. Phys. Rev. Lett. 2008, 101, 157601.

22. Forzatti, P.; Lietti, L. Catal. Today 1999, 52, 165-181.

23. Dil, H.; Lobo-Checa, J.; Laskowski, R.; Blaha, P.; Berner, S.; Osterwalder, J.; Greber, T. Science 2008, 319, 18241826.

24. Avouris, P.; Chen, Z.; Perebeinos, V. Nat. Nanotechnol. 2007, 2, 605-615.

25. Wu, Z.-S.; Pei, S.; Ren, W.; Tang, D.; Gao, L.; Liu, B.; Li, F.; Liu, C.; Cheng, H.-M. Adv. Mater. 2009, 21, 1756-1760. 26. Swart, J. C. W.; van Steen, E.; Ciobica, I. M.; van Santen, R. A. Phys. Chem. Chem. Phys. 2009, 11, 803-807.

27. Ruffieux, P.; Ait-Mansour, K.; Bendounan, A.; Fasel, R.; Patthey, L.; Gröning, P.; Gröning, O. Phys. Rev. Lett. 2009, 102, 086807.

28. Ploigt, H.-C.; Brun, C.; Pivetta, M.; Patthey, F.; Schneider, W.-D. Phys. Rev. B 2007, 76, 195404.

29. van Hardeveld, R. M.; van Santen, R. A.; Niemantsverdriet, J. W. Surf. Sci. 1996, 369, 23-35.

30. Kubby, J. A.; Wang, Y. R.; Greene, W. J. Phys. Rev. B 1991, 43, 9346-9349.

31. Lin, C. L.; Lu, S. M.; Su, W. B.; Shih, H. T.; Wu, B. F.; Yao, Y. D.; Chang, C. S.; Tsong, T. T. Phys. Rev. Lett. 2007, 99 216103.

32. Giovannetti, G.; Khomyakov, P. A.; Brocks, G.; Karpan, V. M.; van den Brink, J.; Kelly, P. J. Phys. Rev. Lett. 2008, $101,026803$.

33. Su, W. S.; Leung, T. C.; Chan, C. T. Phys. Rev. B 2007, 76, 235413.

34. Price, P. J. Phys. Rev. B 1988, 38, 1994-1998.

35. Zhang, H. G.; Hu, H.; Pan, Y.; Mao, J. H.; Gao, M.; Guo, H. M.; Du, S. X.; Greber, T.; Gao, H.-J. J. Phys.: Condens. Matter 2010, 22, 302001.

36. Jiang, D. E.; Du, M.-H.; Dai, S. J. Chem. Phys. 2009, 130 074705 .

37. Wang, B.; Bocquet, M.-L.; Marchini, S.; Gunther, S.; Wintterlin, J. Phys. Chem. Chem. Phys. 2008, 10, 35303534.

38. Michaelides, A.; Hu, P.; Lee, M.-H.; Alavi, A.; King, D. A. Phys. Rev. Lett. 2003, 90, 246103.

39. Sutter, P.; Sadowski, J. T.; Sutter, E. Phys. Rev. B 2009, 80,245411

40. Lahiri, J.; Lin, Y.; Bozkurt, P.; Oleynik, I. I.; Batzill, M. Nat. Nanotechnol. 2010, 5, 326-329.

41. Fuentes-Cabrera, M.; Baskes, M. I.; Melechko, A. V.; Simpson, M. L. Phys. Rev. B 2008, 77, 035405.
42. Vanin, M.; Mortensen, J. J.; Kelkkanen, A. K.; GarciaLastra, J. M.; Thygesen, K. S.; Jacobsen, K. W. Phys. Rev. B 2010, 81, 081408 .

43. Hammer, B.; Nørskov, J. K. Adv. Catal. 2000, 45, 71.

44. Kresse, G.; Furthmüller, J. Phys. Rev. B 1996, 54, 1116911186.

45. Kresse, G.; Hafner, J. Phys. Rev. B 1993, 47, 558-561.

46. Kresse, G.; Hafner, J. Phys. Rev. B 1994, 49, 1425114269.

47. Perdew, J. P.; Wang, Y. Phys. Rev. B 1992, 45, 1324413249.

48. Blöchl, P. E. Phys. Rev. B 1994, 50, 17953-17979. 